PAPERS

\title{
Real time optimization of temperature field in induction heating
}

\author{
Zdeněk Novák, Jan Kyncl ${ }^{*}$
}

\begin{abstract}
Many induction heating processes must be controlled in accordance with the prescribed time evolution of temperature of the heated body (bodies). This requires a correct setting of field currents (amplitudes and frequencies) in the heating inductors that can vary either continuously or by steps. The paper presents a novel model of induction annealing of cylindrical aluminum billets based on solution of nonstationary forward and inverse tasks. The methodology is described in detail and illustrated with a typical example. Some of the results were verified experimentally.

K e y w ords: induction heating, temperature field optimization, annealing, mathematical modelling
\end{abstract}

\section{Introduction}

Thermal processing of metals includes all technologies for their heating and cooling in order to reach the required properties. Reaching of the balance state in the course of phase changes in solid state is determined by diffusion and this process has the decisive influence on the result of the heat treatment. According to influencing the diffusion, thermal processing may be divided into two groups: annealing and hardening. In case of annealing, the diffusion is either supported or only weakly decelerated, while hardening is characterized by its strong damping or the diffusion is completely suppressed. Every thermal processing consists of three operations: heating to the required temperature, equalization of temperatures where necessary and cooling to the final temperature.

This paper solves an inverse task: proposal of heating the cylindrical billet with the requirement of the final temperature profile $T\left(t, t_{\max }\right)$ in the end of the process. The principal advantage of induction heating $(\mathrm{IH})$ is to reach such a profile by a change of the boundary condition or amplitude and frequency of the field current. One can achieve the required profile using the optimization methods. Here, evaluation of the objective function necessitates a series of solutions of the electromagnetic and temperature fields that represent the most demanding part of the algorithm. Every temperature field is, however, really achievable and application of the developed algorithms will be demonstrated only on real processes corresponding to typical technological operations.

Although the problem is solved as linear from the viewpoint of electromagnetic field, the chosen procedure is applicable to non-linear problems including ferromagnetics. This procedure was chosen because the linear problem can be solved using conventional real-time computing. In the case of ferromagnetics, the computational complexity is considerably higher, however, nothing would change in the proposed optimization principle of solution of the inverse problem.

\section{Mathematical models}

The forward task generally represents a triply coupled problem characterized by a mutual interaction of three physical fields: electromagnetic field, temperature field and field of flow. Harmonic electromagnetic field is described by $1 \mathrm{D}$ wave equations in the cylindrical coordinates [1]

$$
\begin{gathered}
\frac{\partial^{2} H_{z}}{\partial r^{2}}+\frac{1}{r} \frac{\partial H_{z}}{\partial r}-j \omega \gamma \mu H_{z}=0 \\
\frac{\partial^{2} E_{\varphi}}{\partial r^{2}}+\frac{1}{r} \frac{\partial E_{\varphi}}{\partial r}-j \omega \gamma \mu E_{\varphi}-\frac{E_{\varphi}}{r^{2}}=0 .
\end{gathered}
$$

Here, $H_{z}(r)$ denotes the axial component of the phasor of magnetic field strength, $E_{\varphi}(r)$ is the azimuthal component of the phasor of electric field strength, $\omega$ denotes the angular frequency, $\gamma$ stands for the electric conductivity, $\mu$ represents the magnetic permeability and $r$ is the radius. The boundary conditions are

$$
E_{\varphi}(0)=0, \quad H_{z}\left(r_{2}\right)=N I / l,
$$

where $r_{2}$ is radius of the cylinder, $N$ is number of turns and $l$ is the length of inductor and $I$ is the current RMS value. The distribution of temperature field is described by the heat transfer equation [2]

$$
\rho c_{p}(T) \frac{\partial T(r, t)}{\partial t}=\operatorname{div}(\lambda(T) \operatorname{grad} T)+w
$$

with the initial condition

$$
T\left(r, t_{\mathrm{st}}\right)=T_{0}
$$

*Department of Electrical Power Engineering, Faculty of Electrical Engineering, CTU Prague, Czech Republic, zdenek.novak@fel.cvut.cz, kyncl@fel.cvut.cz

DOI: $10.2478 /$ jee-2019-0070, Print (till 2015) ISSN 1335-3632, On-line ISSN 1339-309X

(C) This is an open access article licensed under the Creative Commons Attribution-NonCommercial-NoDerivs License (http://creativecommons.org/licenses/by-nc-nd/3.0/). 
Table 1. Values of $c$ and $n$ for (11)

\begin{tabular}{lcc}
\hline$G r P r$ & $c$ & $n$ \\
\hline $1 \times 10^{-3}-5 \times 10^{3}$ & 1.18 & $1 / 8$ \\
$5 \times 10^{3}-2 \times 10^{7}$ & 0.54 & $1 / 4$ \\
$2 \times 10^{7}-1 \times 10^{13}$ & 0.135 & $1 / 3$ \\
\hline
\end{tabular}

$$
\begin{gathered}
\lim _{r \rightarrow 0} \frac{\partial T(r, t)}{\partial r}=0 \\
-\lambda(T) \frac{\partial T\left(r_{2}, t\right)}{\partial r}=\alpha\left(T\left(r_{2}, t\right)-T_{0}\right)+\epsilon \sigma\left(T\left(r_{2}, t\right)^{4}-T_{0}^{4}\right)
\end{gathered}
$$

Symbol $w$ denotes the volumetric Joule loss, $\alpha$ is the heat transfer coefficient describing the convection of the heat transfer, $\sigma$ is the Stefan-Boltzmann constant and is the emissivity

$$
w=\gamma|E(r)|^{2}, \quad \alpha=\frac{N u \lambda}{D}
$$

were, the coefficient $\alpha$ may be expressed in terms of the Nusselt number $N u$ and $D$ denotes the characteristic length of the investigated system. The material parameters $\gamma(T), \lambda(T)$ and $\rho c_{p}(T)$ are in general functions of temperature. The flow field in the vicinity of the cylinder affects the heat transfer convective coefficient $\alpha$ appearing in (8).

Numerically, the influence of convection can be solved only in very simple cases. That is why the convection of heat between the heated surface and fluid is solved mostly experimentally and the results may be generalized using the similarity theory, [2],

$$
N u=c(G r P r)^{n},
$$

where the constants $c$ and $n$ are functions of the product $G r \operatorname{Pr} G r$ - being the Grashof number and $\operatorname{Pr}$ the Prandtl numbers, see Tab. 1.

The Grashof number follows from the comparison of buoyancy forces and friction forces in fluid and may be expressed as

$$
G r=\beta \Delta T\left(g D^{3}\right) / \nu^{2},
$$

where, $\beta$ is the coefficient of cubic dilatation of fluid, $\Delta T$ denotes the difference of temperatures between the fluid and distant environment, $g$ stands for the gravitational acceleration, $D$ is the diameter of the cylinder and $\nu$ represents the kinematic viscosity. Finally, the Prandtl number is given by the formula

$$
\operatorname{Pr}=\left(\eta c_{p}\right) / \lambda
$$

where $\eta$ denotes the dynamic viscosity.

\subsection{Forward problem}

The numerical solution of partial differential equations (1), (2) and (5) is demanding and time-consuming and unsuitable for evaluation in the backward task, mainly if the computations are intended for an on-line control. That is why these equations are transformed to a system of $n$ DAE equations. If we divide the cylinder along its radius to $n$ segments according to Fig. 1, where every segment $\left(r_{i}\right)$ is influenced by the external segment $\left(r_{i+1}\right)$ and internal segment $\left(r_{i-1}\right)$.

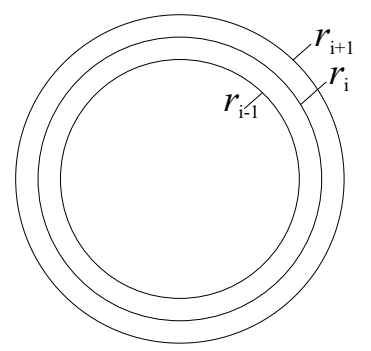

Fig. 1. Segment $r_{i}$ and its neighboring segments $r_{i-1}$ and $r_{i+1}$

Equation (5) may be rewritten as a system of equations

$$
\begin{gathered}
m(i) c_{p} \frac{\partial T_{i}(t)}{\partial t}=\frac{T_{i-1}(t)-T_{i}(t)}{R_{\vartheta}\left(r_{i}, r_{i-1}\right)}+ \\
\frac{T_{i+1}(t)-T_{i}(t)}{\left.R_{\vartheta}\left(r_{i+1}, r_{i}\right)\right)}+P(i),
\end{gathered}
$$

where $i$ is the position of the segment in the cylinder, the last term in (14) (power) is $P(i)=V(i) w\left(r_{i}\right)$, and further

$$
\begin{gathered}
R_{\vartheta}\left(r_{a}, r_{b}\right)=\frac{1}{2 \pi l \lambda} \ln \left(\frac{r_{a}}{r_{b}}\right), \\
V(i)=\frac{l \pi}{2}\left(r_{i+1}^{2}-r_{i-1}^{2}\right) .
\end{gathered}
$$

The marginal segments must include the boundary conditions

$$
\begin{gathered}
T_{1}(t)=T_{2}(t) \\
\frac{T_{n-1}(t)-T_{n}(t)}{R_{\vartheta}\left(r_{n}, r_{n-1}\right)}=\pi r_{n}\left(\alpha\left(T_{n}-T_{0}\right)+\sigma \epsilon\left(T_{n}^{4}-T_{0}^{4}\right)\right) .
\end{gathered}
$$

\subsection{Backward problem}

The problem was solved by several optimization algorithms, both deterministic (gradient and NelderMead methods) and heuristic (Differential evolution method and Simulated annealing method). Particular results will be discussed in the section Illustrative example. The objective function was chosen as

$$
\sum_{i}\left(T_{\text {ent }}\left(r_{i}\right)-T_{\text {cal }}\left(r_{i}\right)\right)^{2},
$$

where, $T_{\text {ent }}$ is the required temperature and $T_{\text {cal }}$ is the calculated temperature.

Gradient method uses computation or estimation of the function derivative. For the functions of one variable we use the Newton method. Its principle is based on the construction of the tangent derivative of the function $f^{\prime}(x)$ at $\left(x_{k}, f^{\prime}\left(x_{k}\right)\right)$. The following $k+1$ iteration of the 


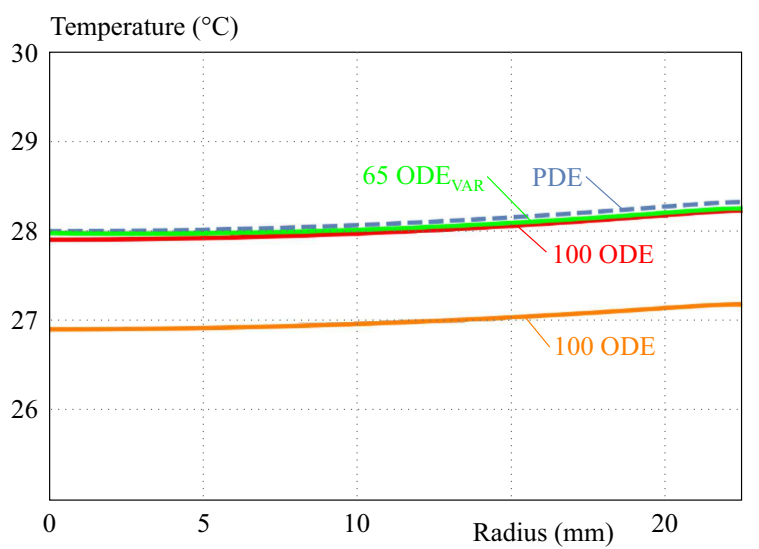

Fig. 2. Comparison of results of temperature field using PDE and ODE equation systems

optimum point $x_{\mathrm{opt}}$ is the intersection of this tangent with the $x$-axis. The details of this method can be found in $[3-5]$.

Nelder-Mead method is a direct search method. For a function with $n$ variables, the algorithm maintains the set of $n+1$ points forming the polytope peaks in an $n$ dimensional space. This method is often called the "simplex" method, which should not be confused with the known simplex method for linear programming. For details see [6-7].

Differential evolution is a simple stochastic function minimizer. The algorithm maintains a population of $m$ points, $x_{1}, x_{2}, \ldots, x_{j}, \ldots, x_{m}$, where typically $m>>n$, $n$ are the number of variables. A new $m$-point population is generated during every algorithm iteration. The new point $j$ is generated by selecting three random points, $x_{u}$, $x_{v}$ and $x_{w}$ from the old population and creating $x_{s}=$ $s_{w}+s\left(x_{u}-x_{v}\right)$, where $s$ is the actual scale factor. Then a new point $x_{\text {new }}$ is created from $x_{j}$ and $x_{s}$ by taking the coordinate $i$ from $x_{s}$ with the probability $\rho$ and otherwise taking the coordinate from $x_{j}$. If $f\left(x_{\text {new }}\right)<$ $f\left(x_{j}\right)$, then $x_{\text {new }}$ replaces $x_{j}$ in the population. More detail can be found in [8-10].

Simulated annealing is a simple stochastic function minimizer. It is motivated by the physical annealing process where the metal object is heated to a high temperature and allowed to cool slowly. The process allows settling the atomic structure of the metal in a lower energy state, thus making a harder metal. Utilizing optimization terminology, annealing allows the structure to escape from the local minima, and explore and settle on a better, perhaps global minimum. Details can be found in [11-13].

\section{Numerical solution}

The main computations are carried out as a coupled problem in $1 \mathrm{D}$ in the central part of the cylinder, where the thermal transfer is not influenced by the front effects in its lower and upper parts. The results are checked by numerical computations in $2 \mathrm{D}$ carried out by the finite element method.

\subsection{Forward problem}

The numerical solution was performed using SW Wolfram Mathematica. The aim of this chapter is to compare selected variants of the task performed by solving partial differential equation and a system of ordinary differential equations (ODEs). For the system of ODEs, it is necessary to choose the optimal number of segments dividing the heated cylinder as shown in Fig. 2. A low number of segments leads to higher inaccuracies in the results and, vice versa, a higher number of segments substantially prolongs the computations.

The comparison for resultant temperature distribution was performed for current of $160 \mathrm{~A}$, frequency of $21 \mathrm{kHz}$ and time of $120 \mathrm{~s}$. The essential point was choosing the constant step $\Delta r$. It holds $\Delta r=r /(n-1)$, where $r$ is the radius of the heated cylinder and $n$ is the number of segments. The computation parameters for particular methods and the temperature distributions are given in Tab. 2 and in Fig. 2.

As can be seen from Fig. 2, with growing number of segments the accuracy of results grows, but at the expense of the computation time. To find the optimal number of segments, we will require that the accuracy of the system of ODEs would be quite comparable with PDE, but at as low computation demands as possible.

Evaluation of the volumetric heat power according to (9) we find that this value abruptly decreases with multiples of the depth of penetration. That is why the situation in the surface layers is much more important than near the axis of symmetry.

Table 2. Calculation times of individual methods

\begin{tabular}{lc}
\hline PDE & 4.520 \\
\hline ODE 1000 & 0.450 \\
ODE 100 & 0.040 \\
ODEVAR 65 & 0.024 \\
\hline
\end{tabular}

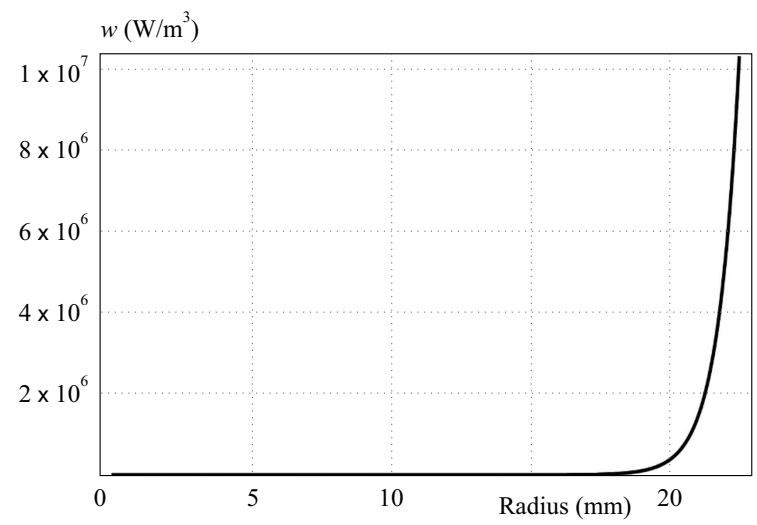

Fig. 3. Quantifying the power density w in cylinder for $I=160$ A and $f=21 \mathrm{kHz}$ 
The computations started with testing segments of nonhomogeneous widths, where radii are given by the expression

$$
r_{i}=a+\frac{b}{i}+c \ln i
$$

where $a, b, c$ are constants and $i \in N$.

For $n=200$ the constants are: $a=5.613 \times 10^{-3}$, $b=5.603 \times 10^{-3}$ and $c=3.192 \times 10^{-3}$, and for $n=65$ the constants are: $a=5.583 \times 10^{-3}, b=5.573 \times 10^{-3}$ and $c=4.073 \times 10^{-3}$

As is obvious from Tab. 2 and graph in Fig. 2, this distribution is not only highly accurate, but the solution is also very fast.

\subsection{Backward problem}

The temperature profile was chosen from the forward problem to verify the optimization methods on some illustrative examples. The aim is to select and verify the optimization method, which will provide the fastest possible solution with an adequate precision. For comparison, the following methods were chosen: Gradient method, NelderMead method, Differential evolution method, and Simulated annealing. Each of the tested methods has a graphical representation of the convergence on the number of steps and a graphical representation of the error on the cylinder radius. In Tab. 3, all methods and their minimum search times, the number of steps and the highest error are clearly shown. The test for all methods was similar, it was necessary to find $\mathrm{n}$ currents and $\mathrm{n}$ frequencies. Already for $n=4$ the temperature curve corresponds to the predetermined curve.

The first tested method was the gradient method. As can be seen from Fig. 4 it finds the setpoint after a few steps, but it still tries to find the correct value and after 499 steps the algorithm ends and evaluates the smallest minimum it has calculated. The deviation does not exceed $10^{-1} \mathrm{~K}$ from the search temperature curve for the founded current and frequency.

The second, Nelder-Mead method was tested in two variants, with and without Random Seed. As shown in Fig. 5, for this method it is enough to perform much fewer steps than above to find the most accurate value, but for

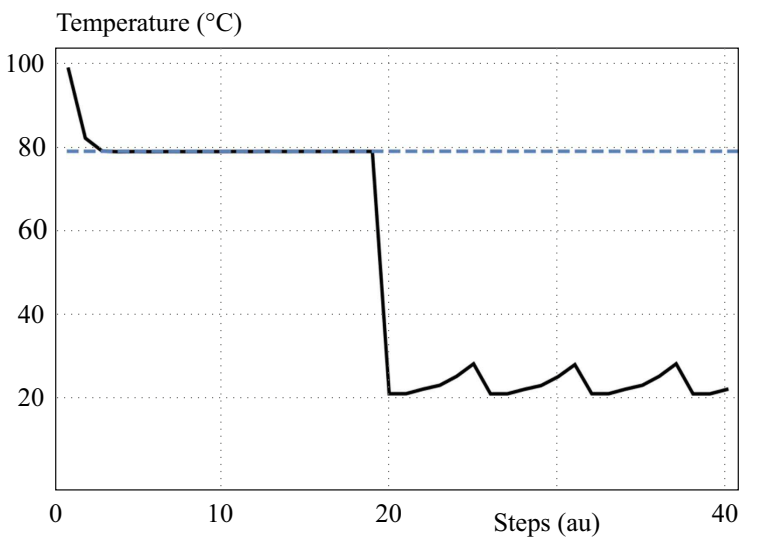

Fig. 4. Evolution of temperature in each step for the gradient method for first 40 steps the variant with Random Seed=100 the number of steps is higher (Fig. 6). The deviation is negligibly small from the search temperature curve for the founded current and frequency.

The third method tested is Differential evolution. Figure 7 show that this is the most accurate method, but it requires a higher number of steps. The deviation is negligibly small from the search temperature curve for the founded current and frequency.

The last method was simulated annealing. It is obvious that the optimum is searched for in a large number of steps (Fig. 8). The deviation from the search temperature curve for the founded current and frequency is negligibly small.

Testing individual methods was carried out to evaluate the fastest method to optimize the source as described in the chapter Examples. The methods are summarized in Tab. 3. It is evident that Differential evolution is the slowest method (though not the highest number of steps). This is not surprising; this method is the most accurate but unusable to use for resource optimization. Similarly, the Simulated annealing method did not work, although its calculation time is not if that of Differential evolution, but it is still far from being usable. Nelder-Mead for either option cannot be used, the calculation time exceeds one minute, which is for on-line control also unusable. Despite many steps, the most economical and ideal for our applications is the Gradient method.

Table 3. Compare methods to the number of steps and time

\begin{tabular}{lcc}
\hline Method & Steps & Solution time \\
\hline Gradient & 499 & 29 \\
Nelder-Mead & 43 & 85 \\
Nelder-Mead with random seed & 54 & 65 \\
Differential evolution & 95 & 1082 \\
Simulated annealing & 808 & 396 \\
\hline
\end{tabular}

\section{Examples}

The presented examples will illustrate the advantages of the methodology. The measurements were realized us-

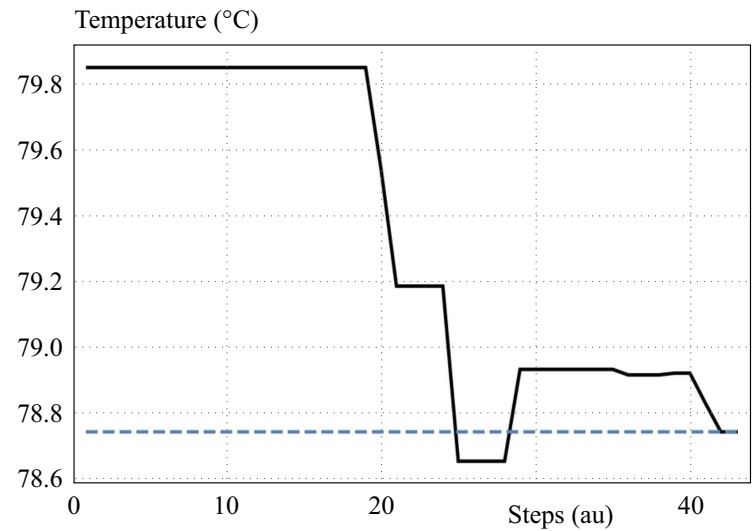

Fig. 5. Evolution of temperature in every step for nelder-mead method without random seed 
Temperature $\left({ }^{\circ} \mathrm{C}\right)$

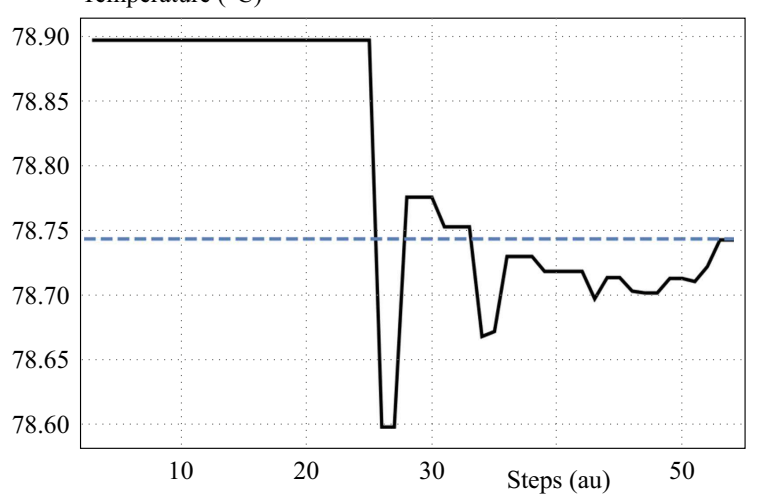

Fig. 6. Evolution of temperature in every step of nelder-mead method with random seed $=100$

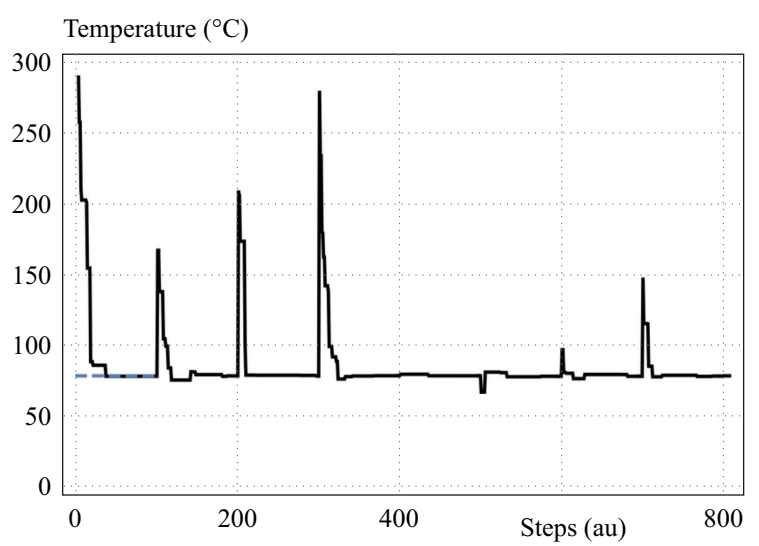

Fig. 8. Evolution of temperature in every step for simulated annealing method

ing thermo-camera and capturing amplitudes and frequencies of the current delivered to the inductor.

The search for the optimal curve is divided into $n$ steps in which the current $I$ and the frequency $f$ are searched. The whole process works by calculating all $n$ values of $I$ and $f$ in the $i$-th time step and re-setting the $i$-th value at the source. Every $i$-th step is refined by measur-

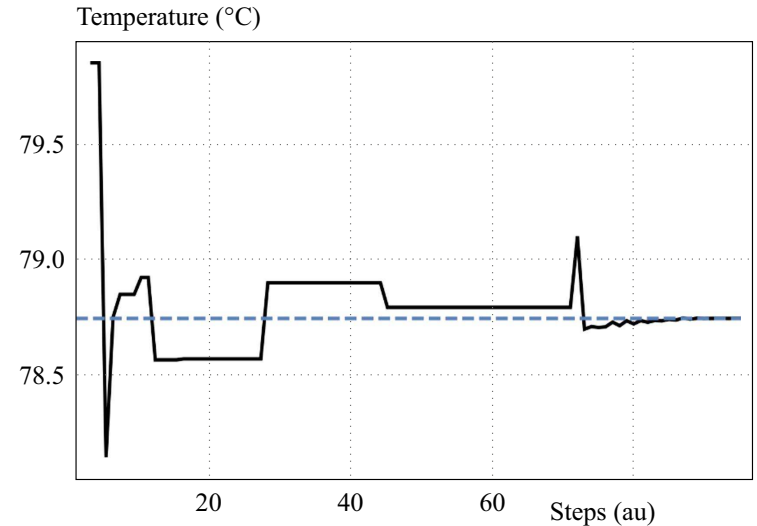

Fig. 7. Evolution of temperature in every step for differential evolution method

ing from a thermal imager where direct measurement in the $i$-th step serves as a new boundary condition of the temperature on the cylinder radius. In the next step, only the $n-1$ remaining steps are already counted because previous $i$ values are already set, as the time-consuming optimization is less. The algorithm continues until the time when $n-i=0$. The algorithm ends, and we have the required temperature curve. The flowchart is depicted in Fig. 9.

\subsection{Power source}

The whole experiment was carried out on a roll ingot of diameter $d=45 \mathrm{~mm}$ and length $l=100 \mathrm{~mm}$, which was inserted into an inductor fed from harmonic current source. Material properties of AW-2030 aluminum are thermal conductivity $\lambda=130 \mathrm{~W} / \mathrm{mK}$, density $\rho=$ $2810 \mathrm{~kg} / \mathrm{m}^{3}$ and specific heat capacity $c_{\mathrm{p}}=860 \mathrm{~J} / \mathrm{kgK}$. The ingot itself was stained by a special thermographic spray paint with high emissivity LabIR Paint.

When the ingot has a defined temperature, we can scan it using a thermal imager (Fig. 11), which we use for the new initial temperatures in the calculation system according to the flowchart (Fig. 9).

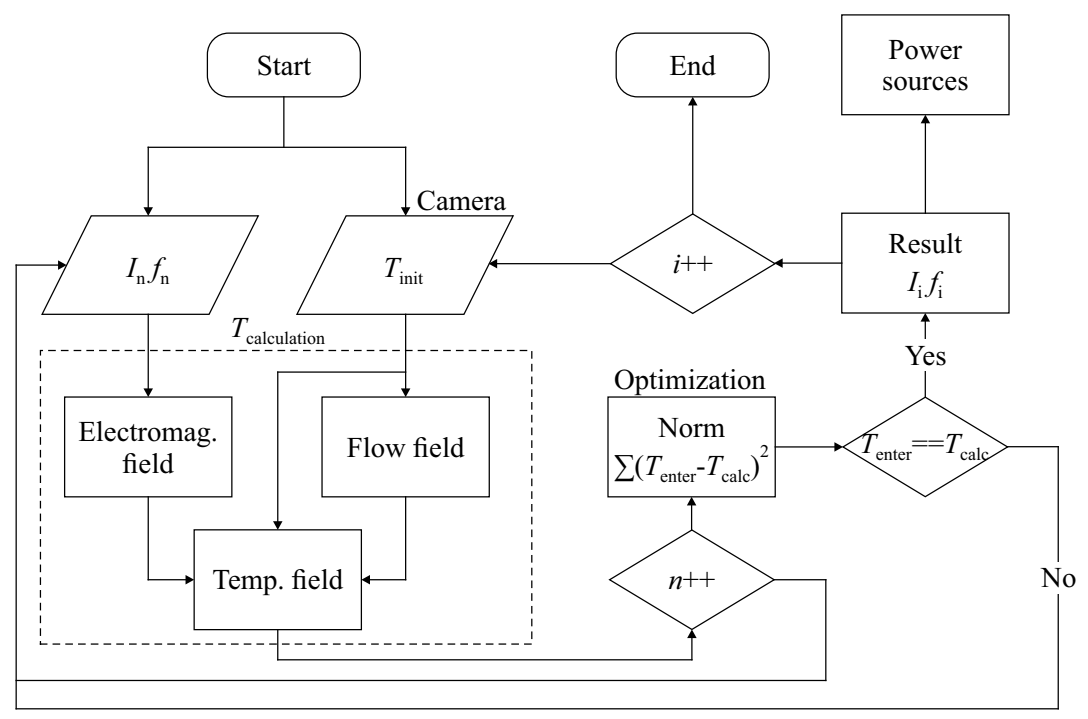

Fig. 9. Algorithm of entire process 


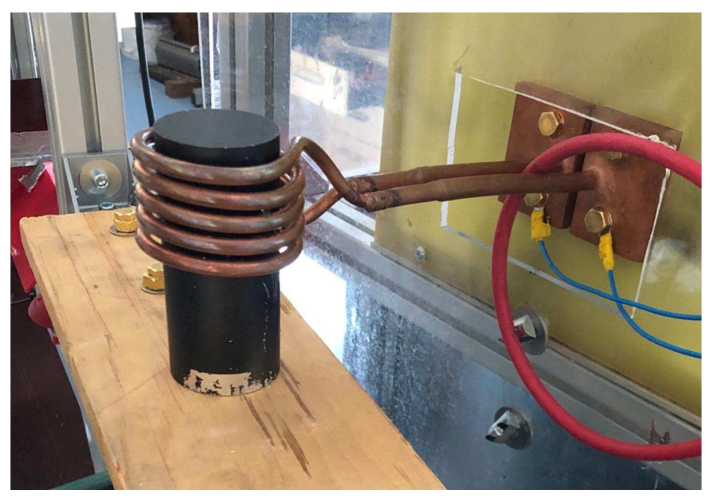

Fig. 10. View of inductor and charge

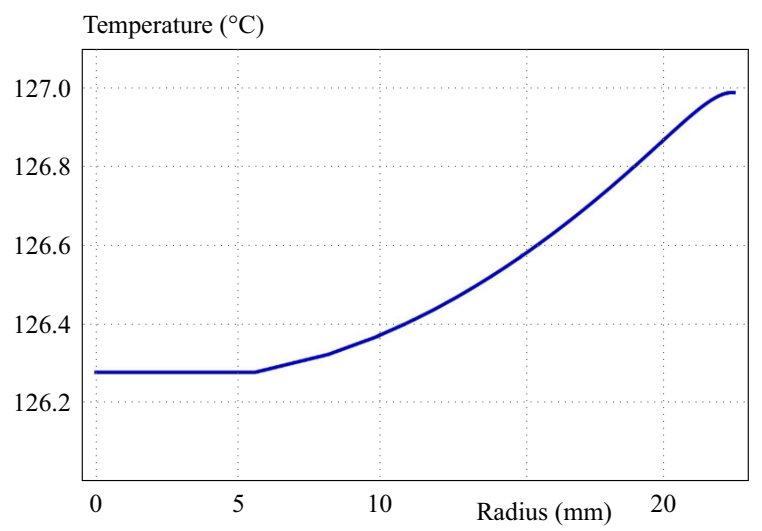

Fig. 12. Required temperature dependence on cylinder radius

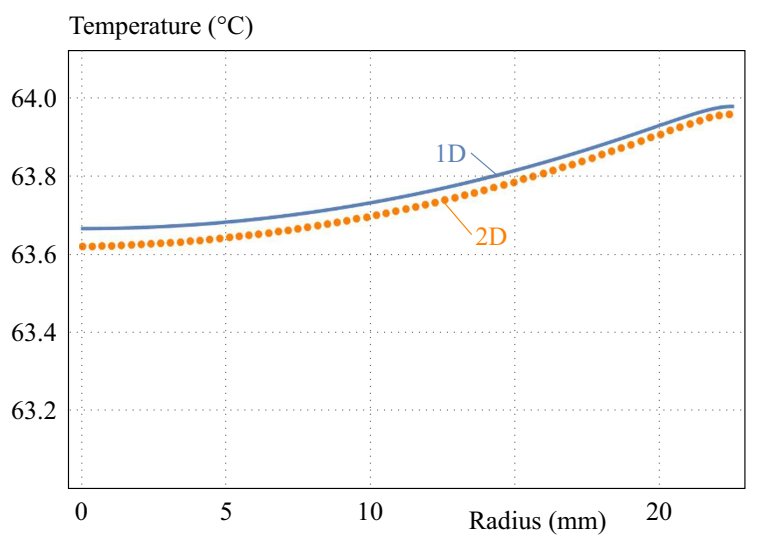

Fig. 14. Comparison of selected $1 \mathrm{D}$ and $2 \mathrm{D}$ results

\subsection{Results of experiment}

Power source parameters allow continuous streaming but discontinuous frequency adjustment. This is due to the resonant circuit. Therefore, the optimization process also searches for continuous current values and discontinuous frequency values. Currents were searched for $I_{\max }=500 \mathrm{~A}$ and several frequencies. Figure 12 shows the distribution of temperature along the radius of an aluminum cylinder for time $t=174 \mathrm{~s}$ in $n=4$ times steps and frequency $21.5 \mathrm{kHz}$.

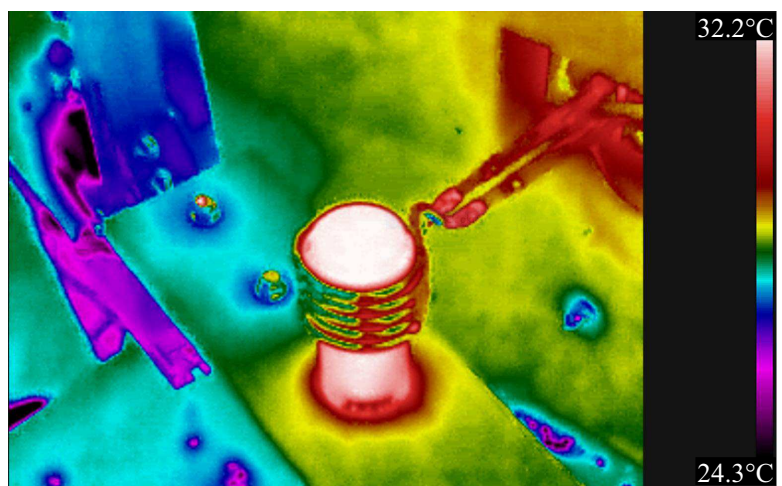

Fig. 11. Thermographic measurement

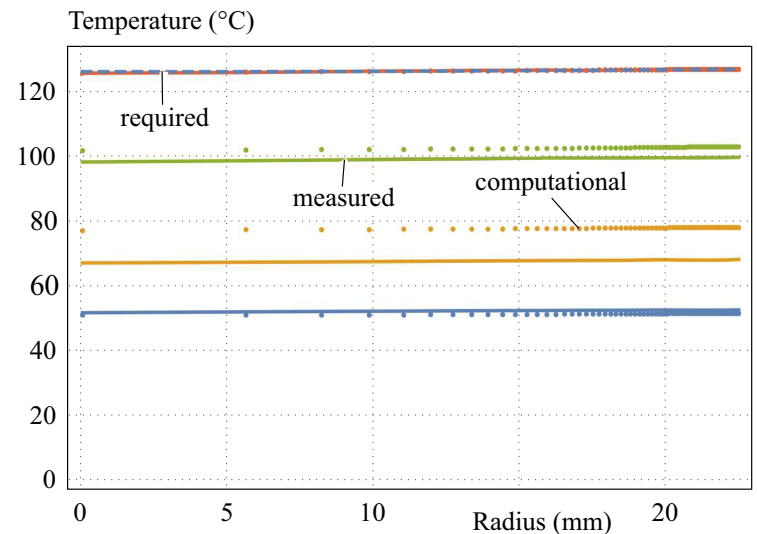

Fig. 13. Step-by-step optimization results for gradient method

Table 4. Currents in Ampers in each step for tested methods

\begin{tabular}{lcccc}
\hline Method & $I_{1}$ & $I_{2}$ & $I_{3}$ & $I_{4}$ \\
\hline Gradient & 148.9 & 155.5 & 216.9 & 205.9 \\
Nelder-Mead & 151.4 & 169.5 & 216.8 & 205.9 \\
Differential evolution & 180.0 & 177.8 & 216.9 & 205.9 \\
Simulated annealing & 138.9 & 168.5 & 216.9 & 205.9 \\
\hline
\end{tabular}

The results of currents for all tested methods are given in Tab. 4. The results of frequencies were for all the steps $f=21.5 \mathrm{kHz}$.

The results of the gradient method are in Fig. 13 and show the development of the calculated (dotted lines) and measured (solid lines) temperatures in each step. It is evident that the curve merges in the last step. The following methods are slower, but they demonstrate almost the same result and differ only in the first two steps.

The selection of the optimization method in our case is not related to accuracy, due to similar results especially in the last two steps. It is related to the speed of the calculation, because of the need for online measurement and power source setting. The fastest method is the gradient method tested as the first one.

\section{$4.32 D$ model}

The simplification we used for the 1D model for faster optimization is confirmed by a comparison with the $2 \mathrm{D}$ 


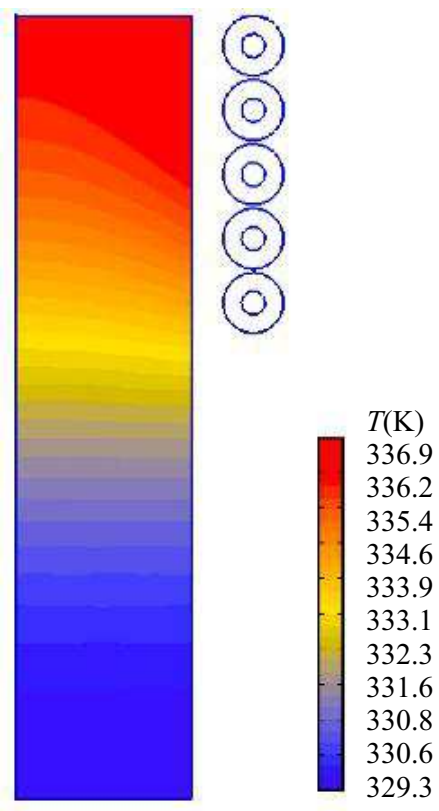

Fig. 15. 2D FEM result

solution realized by FEM SW Agros2D. The temperature is measured by a thermal imager at the upper edge, so this temperature will be scanned from the same edge in the 2D model and compared to $1 \mathrm{D}$.

The objects are a cylinder inside a 5-turn inductor. These objects are rotationally symmetrical bodies, so it is best to draw these bodies in axially symmetric mode. The inductor, like the experiment, is stored with the batch edge. Solver SW Agros2D is set to solve transient phenomena with the same simulation time for comparison with the 1D problem. Figure 14 shows the corresponding graphs. The 2D FEM model results are closer to the 1D model result, so we can use the faster 1D field method.

\section{Conclusion}

The paper presents a complete mathematical model of induction heating for the possibility of inverse optimization method in the course of quenching or annealing of a metal cylinder. As the results practically agree with the results obtained by $2 \mathrm{D}$ computations realized by the finite element method, the 1D model can be preferred for simpler geometries.

A number of optimization methods have been investigated. The first result was the time demand of particular methods. Secondly, it was a real-time solution when using new thermal imaging conditions. The disadvantage is that the simulation time still exceeds the real heating time.

However, this document demonstrates that this optimization method can be developed for some applications. Future research will be conducted to optimize other physical fields (e.g. field of thermoelastic displacement). Then, more complex geometrical configurations will be studied.
Optionally, the described methodology will be extended to heating by multiple frequencies at the same time.

\section{Acknowledgement}

This work was supported by the Department of Electrical Power Engineering CTU-FEE.

\section{REFERENCES}

[1] G. Lehner and M. Horrer, Electromagnetic Field Theory for Engineers Physicists Springer Berlin, 2014.

[2] J. Lienhard, A Heat Transfer Textbook, Dover Publications, 2013.

[3] Y. Song and L. Liu, "A Nonmonotone Conjugate Gradient Method for Nonlinear Optimization, Third International Joint Conference on Computational Science Optimization, pp. 132135, 2010.

[4] M. Cao and Y. Yang, "Two Classes of Conjugate Gradient Methods for Large-Scale Unconstrained Optimization, 2011 Fourth International Joint Conference on Computational Sciences Optimization, pp. 3740, 2011.

[5] D. Fujian and S. Zhongbo, "A modified LiuStorey conjugate gradient method its global convergence for unconstrained optimization, 2010 Chinese Control Decision Conference,pp. 15851588, 2010.

[6] N. Citroen, M. Ouassaid, and M. Maaroufi, "Optimization of the subscribed maximum demand for the high voltage tariff consumers in Morocco using Nelder Mead method, 3rd International Renewable Sustainable Energy Conference IRSEC, pp. 16, 2015.

[7] K. Chang and H. Lu, "Solving quantile-based stochastic optimization problems with modified Stochastic Nelder-Mead Simplex Method, Proceedings of the 2014 IEEE 18th International Conference on Computer Supported Cooperative Work in Design CSCWD, pp. 374379, 2014.

[8] D. Ustun and A. Akdagli, "A study on the performance of the hybrid optimization method based on artificial bee colony differential evolution algorithms, International Artificial Intelligence Data Processing Symposium IDAP, pp. 15, 2017.

[9] C. Wu and K. Tseng, "Engineering Optimization Using Modified Binary Differential Evolution Algorithm, Third International Joint Conference on Computational Science Optimization, pp. 501505, 2010.

[10] L. B. Zhang, X. L. Xu, C. T. Sun, and C. G. Zhou, "A Modified Differential Evolution Multi-objective Optimization Method, International Conference on Computational Intelligence Natural Computing, pp. 511514, 2009.

11] G. Wang, C. Xu, and G. Liu, "The transient electromagnetic inversion based on the simplex-simulated annealing algorithm, 37th Chinese Control Conference CCC, pp. 43214324, 2018.

[12] M. Gao and J. Tian, "Network Intrusion Detection Method Based on Improved Simulated Annealing Neural Network, International Conference on Measuring Technology Mechatronics Automation, pp. 261264, 2009.

[13] S. Yan, S. Zheng, and H. Kun, "An Isolation Niche Hybrid Genetic Algorithm Based on Simulated Annealing Method, Chinese Control Conference, pp. 776779, 2006.

Received 6 June 2019

Zdeněk Novák was born 1987. He received the Ing. degree in electrical engineering from the Czech Technical University in Prague, Prague, Czech Republic, in 2012, where he is currently working toward the $\mathrm{PhD}$ degree.

Jan Kyncl is an associated professor of Czech Technical University, born in 1969. His main research areas are mathematical modeling of power engineering systems and electrical heating. 